\title{
Classical Wakimoto Realizations of Chiral WZNW Bloch Waves
}

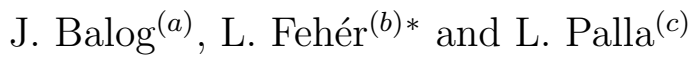 \\ ${ }^{(a)}$ Research Institute for Nuclear and Particle Physics, \\ Hungarian Academy of Sciences, \\ H-1525 Budapest 114, P.O.B. 49, Hungary \\ ${ }^{(b)}$ Institute for Theoretical Physics, József Attila University, \\ H-6726 Szeged, Tisza Lajos krt 84-86, Hungary \\ (c) Institute for Theoretical Physics, Roland Eötvös University, \\ H-1117, Budapest, Pázmány P. sétány 1 A-ép, Hungary
}

\begin{abstract}
It is well-known that the chiral WZNW Bloch waves satisfy a quadratic classical exchange algebra which implies the affine Kac-Moody algebra for the corresponding currents. We here obtain a direct derivation of the exchange algebra by inverting the symplectic form on the space of Bloch waves, and give a completely algorithmic construction of its generalized free field realizations that extend the classical Wakimoto realizations of the current algebra.
\end{abstract}

PACS codes: 11.25.Hf, 11.10.Kk, 11.30.Na

keywords: WZNW model, exchange algebra, Wakimoto realizations

* Corresponding author's e-mail: lfeher@sol.cc.u-szeged.hu, phone/fax: (+36) 62544368. 


\section{Introduction}

The Wess-Zumino-Novikov-Witten (WZNW) model [1] of conformal field theory has proved to be the source of interesting structures that play an increasingly important rôle in theoretical physics and in mathematics [2, 3]. Among these structures are the quadratic Poisson bracket algebras [4]- [16] that arise from the chiral separation of the degrees of freedom in the model, which is based on the form of the classical solution of the field equation given by

$$
g\left(x_{L}, x_{R}\right)=g_{L}\left(x_{L}\right) g_{R}^{-1}\left(x_{R}\right)
$$

Here $x_{C}(C=L, R)$ are lightcone coordinates and the $g_{C}$ are quasiperiodic group valued fields with equal monodromies, $g_{C}(x+2 \pi)=g_{C}(x) M$ for some $M$ in the WZNW group $G$. The classical exchange algebras can be regarded as fundamental since the affine Kac-Moody (KM) symmetry follows as their consequence, and help to better understand the quantum group properties of the model (see e.g. [17]) by means of canonical quantization [18, 19, 20]. One should however note that after the chiral separation, which essentially amounts to forgetting the equal monodromy constraint on the pair $\left(g_{L}, g_{R}\right)$, the Poisson structure is highly non-unique [14, 15, 16. A natural choice consists in restricting the monodromy $M$ of the chiral WZNW fields to be diagonal, in which case the resulting classical exchange algebra can be described as follows 11, 12, 13. Denoting any of the chiral WZNW 'Bloch waves' by $b(x)$,

$$
b(x+2 \pi)=b(x) e^{\omega}, \quad \omega \in \mathcal{H},
$$

where $\mathcal{H}$ is a Cartan subalgebra of the Lie algebra $\mathcal{G}$ of $G$, the exchange algebra reads as

$$
\begin{gathered}
\{b(x) \stackrel{\otimes}{,} b(y)\}=\frac{1}{\kappa}(b(x) \otimes b(y))\left(\mathcal{R}(\omega)+\frac{1}{2} \hat{I} \operatorname{sign}(y-x)\right), \quad 0<x, y<2 \pi, \\
\mathcal{R}(\omega)=\frac{1}{2} \sum_{\alpha \in \Phi} \operatorname{coth}\left(\frac{1}{2} \alpha(\omega)\right) E_{\alpha} \otimes E^{\alpha}, \quad \hat{I}=E_{\alpha} \otimes E^{\alpha}+H_{k} \otimes H^{k},
\end{gathered}
$$

where $E_{\alpha}, H_{k}$ is a Cartan-Weyl basis of $\mathcal{G}$ and $\kappa$ is a constant. For the 'exchange r-matrix' $\mathcal{R}(\omega)$ to be non-singular, the monodromy parameter $\omega$ has to be restricted to a domain in $\mathcal{H}$ where $\alpha(\omega) \notin i 2 \pi \mathbf{Z}$ for any root $\alpha$. Notice also from (1.3) that the $2 \pi$-periodic current

$$
J=\kappa b^{\prime} b^{-1}
$$

indeed satisfies the KM Poisson brackets

$$
\left\{\operatorname{Tr}\left(T_{a} J\right)(x), \operatorname{Tr}\left(T_{b} J\right)(y)\right\}=\operatorname{Tr}\left(\left[T_{a}, T_{b}\right] J\right)(x) \delta(x-y)+\kappa \operatorname{Tr}\left(T_{a} T_{b}\right) \delta^{\prime}(x-y),
$$

where $\delta(x-y)=\frac{1}{2 \pi} \sum_{n \in \mathbf{Z}} e^{i n(x-y)}$ and $T_{a}$ is any basis of $\mathcal{G}$ (for the notations, see also sec. 2).

Our main purpose here is to construct generalized free field realizations of the exchange algebra given by (1.3) that extend the Wakimoto realizations [21] of the current algebra. The Wakimoto realizations of the KM current are well-understood both at the classical and quantized level and proved useful in many respects (see e.g. [2, 22, 23, 24, 25]). Their extension to accompanying realizations of the WZNW Bloch waves have also received attention [7, 8, 15, 24], but, except for the simplest cases, a fully algorithmic construction has not appeared yet. At 
the classical level, we present such a construction in sec. 3. The construction is summarized by the diagram (3.10), where all the arrows represent Poisson maps, which is a new result for the map $\widehat{W}$ defined by (3.18). For completeness, in sec. 2 we also give a derivation of the exchange algebra. This derivation is quite different from those in [11, 13] and conceptually close to that in [12]. In order to obtain (1.3), we will directly invert the symplectic form - which we use in sec. 3 as well - that the space of Bloch waves inherits from the full WZNW phase space.

\section{Derivation of the exchange algebra of Bloch waves}

Throughout the paper, let $\mathcal{G}$ be either a complex simple Lie algebra or its normal real form, and $G$ a corresponding Lie group. Denote by $\widetilde{G}$ (resp. $\widetilde{\mathcal{G}}$ ) the loop group (algebra) consisting of the $2 \pi$-periodic, smooth, $G$-valued ( $\mathcal{G}$-valued) functions on the real line $\mathbf{R}$. Choose a Cartan subalgebra $\mathcal{H} \subset \mathcal{G}$ that admits the root space decomposition

$$
\mathcal{G}=\mathcal{H} \oplus \sum_{\alpha \in \Phi} \mathcal{G}_{\alpha}
$$

and an associated basis $H_{k} \in \mathcal{H}, E_{\alpha} \in \mathcal{G}_{\alpha}$ normalized by $\operatorname{Tr}\left(E_{\alpha} E_{-\alpha}\right)=\frac{2}{|\alpha|^{2}}$, where $\operatorname{Tr}$ denotes an invariant scalar product on $\mathcal{G}$. By using this basis any $A \in \mathcal{G}$ can be decomposed as

$$
A=A^{0}+A^{r} \quad \text { with } \quad A^{0} \in \mathcal{H}, \quad A^{r}=\sum_{\alpha \in \Phi} E_{\alpha} \operatorname{Tr}\left(E^{\alpha} A\right), \quad E^{\alpha}:=\frac{1}{2}|\alpha|^{2} E_{-\alpha}
$$

Fix an open domain $\mathcal{A} \subset \mathcal{H}$ which has the properties that $\alpha(\omega) \notin i 2 \pi \mathbf{Z}$ for any root, $\alpha \in \Phi \subset$ $\mathcal{H}^{*}$, and the map $\mathcal{A} \ni \omega \mapsto e^{\omega} \in G$ is injective.

Let us now define $\mathcal{M}_{\text {Bloch }}^{G}$ by

$$
\mathcal{M}_{\text {Bloch }}^{G}:=\left\{b \in C^{\infty}(\mathbf{R}, G) \mid b(x+2 \pi)=b(x) e^{\omega}, \quad \omega \in \mathcal{A} \subset \mathcal{H}\right\}
$$

and endow it with the differential 2-form

$$
\Omega_{\text {Bloch }}^{G, \kappa}(b)=-\frac{\kappa}{2} \int_{0}^{2 \pi} d x \operatorname{Tr}\left(b^{-1} d b\right) \wedge\left(b^{-1} d b\right)^{\prime}-\frac{\kappa}{2} \operatorname{Tr}\left(\left(b^{-1} d b\right)(0) \wedge d \omega\right) .
$$

It is easy to check that $d \Omega_{B l o c h}^{G, \kappa}=0$ and below we shall see that this formula actually defines a symplectic form on $\mathcal{M}_{\text {Bloch }}^{G}$. Note that $\Omega_{\text {Bloch }}^{G, \kappa}$ can be obtained from the natural symplectic form on the space of classical solutions of the WZNW model, as explained in [12, 14, 15.

It will be convenient to parametrize $b \in \mathcal{M}_{\text {Bloch }}^{G}$ as

$$
b(x)=h(x) \exp (x \bar{\omega}), \quad \bar{\omega}:=\frac{\omega}{2 \pi},
$$

where $\omega \in \mathcal{A}$ and $h \in \widetilde{G}$. This one-to-one parametrization yields the identification

$$
\mathcal{M}_{\text {Bloch }}^{G}=\widetilde{G} \times \mathcal{A}=\{(h, \omega)\} .
$$

Correspondingly, a vector field $X$ on $\mathcal{M}_{B l o c h}^{G}$ is parametrized by

$$
X=\left(X_{h}, X_{\omega}\right) \quad X_{h} \in T_{h} \widetilde{G} \quad X_{\omega} \in T_{\omega} \mathcal{A} \simeq \mathcal{H}
$$


with $h^{-1} X_{h} \in T_{e} \widetilde{G} \simeq \widetilde{\mathcal{G}}$. By regarding $\omega$ and $h$ as evaluation functions on $\mathcal{M}_{B l o c h}^{G}$, we may write $X_{\omega}=X(\omega)$ and $X_{h}(x)=X(h(x))$. Equivalently, $X$ can be characterized by its action on $b(x)$,

$$
b^{-1}(x) X(b(x))=e^{-x \bar{\omega}} h^{-1}(x) X(h(x)) e^{x \bar{\omega}}+x X(\bar{\omega}),
$$

where the function $b^{-1}(x) X(b(x))$ on $\mathbf{R}$ is uniquely determined by its restriction to $[0,2 \pi]$. In general, the derivative $X(F)$ of a function $F$ on $\mathcal{M}_{B \text { loch }}^{G}$ is defined by using that any vector is the velocity to a smooth curve. That is, if the value of the vector field $X$ at $b \in \mathcal{M}_{B l o c h}^{G}$ coincides with the velocity to the curve $\gamma(x, t)$ at $t=0, \gamma(x, 0)=b(x)$, then for a differentiable function $F$ we have $X(F)[b]=\left.\frac{\mathrm{d}}{\mathrm{d} t} F[\gamma(x, t)]\right|_{t=0}$.

A function $F$ on $\mathcal{M}_{B l o c h}^{G}$ is henceforth called admissible if its derivative with respect to any vector field $X$ exists and has the form

$$
X(F)=\langle d F, X\rangle=\operatorname{Tr}\left(d_{\omega} F X_{\omega}\right)+\int_{0}^{2 \pi} d x \operatorname{Tr}\left(\left(h^{-1} d_{h} F\right)\left(h^{-1} X_{h}\right)\right)
$$

where

$$
d F=\left(d_{h} F, d_{\omega} F\right) \quad \text { with } \quad d_{h} F \in T_{h}^{*} \widetilde{G}, \quad d_{\omega} F \in T_{\omega}^{*} \mathcal{A}
$$

is the exterior derivative of $F$. In this definition we identify $T_{\omega}^{*} \mathcal{A}$ with $\mathcal{H}$ by means of the scalar product "Tr" and also identify $T_{e}^{*} \widetilde{G}$ with $\widetilde{\mathcal{G}}$ by the scalar product $\int_{0}^{2 \pi} \operatorname{Tr}(\cdot, \cdot)$, whereby we have $h^{-1} d_{h} F \in T_{e}^{*} \widetilde{G}=\widetilde{\mathcal{G}}$. It is clear that the local evaluation functions $\mathcal{M}_{\text {Bloch }}^{G} \ni b \mapsto b(x)$ are differentiable but not admissible, while e.g. the Fourier components of the current $J$ and the components of $\omega$ are admissible functions. It is also worth noting that the matrix elements of the nonlocal $G$-valued function given by the path ordered exponential integral of the current $J$ over a period are, in fact, not admissible, but the trace of any of the powers of this (Wilson loop) function is admissible, since it can be expressed as a function of $\omega$ alone.

We now wish to show that $\Omega_{\text {Bloch }}^{G, \kappa}$ is symplectic in the sense that it permits to associate a unique hamiltonian vector field, $Y^{F}$, with any admissible function, $F$. The defining property of $Y^{F}$ is that it must satisfy

$$
\langle d F, X\rangle=X(F)=\Omega_{B l o c h}^{G, \kappa}\left(X, Y^{F}\right)
$$

for any vector field $X$. In order to determine $Y^{F}$ from this equality, we first point out that in terms of the variables $(h, \omega)$

$$
\begin{aligned}
\Omega_{\text {Bloch }}^{G, \kappa}(h, \omega) & =-\frac{\kappa}{2} \int_{0}^{2 \pi} d x \operatorname{Tr}\left(\left(h^{-1} d h\right) \wedge\left(h^{-1} d h\right)^{\prime}\right. \\
& \left.+2 \bar{\omega}\left(h^{-1} d h\right) \wedge\left(h^{-1} d h\right)-2 d \bar{\omega} \wedge h^{-1} d h\right) .
\end{aligned}
$$

It follows that

$$
\begin{aligned}
\Omega_{\text {Bloch }}^{G, \kappa}\left(X, Y^{F}\right) & =-\kappa \int_{0}^{2 \pi} \operatorname{Tr}\left(\left(h^{-1} Y^{F}(h)\right)^{\prime} h^{-1} X(h)+\bar{\omega}\left[h^{-1} X(h), h^{-1} Y^{F}(h)\right]\right. \\
& \left.+Y^{F}(\bar{\omega}) h^{-1} X(h)-X(\bar{\omega}) h^{-1} Y^{F}(h)\right) .
\end{aligned}
$$

This implies the following equations for $Y^{F}$ :

$$
\begin{aligned}
\left(h^{-1} Y^{F}(h)\right)^{\prime}+\left[h^{-1} Y^{F}(h), \bar{\omega}\right]+Y^{F}(\bar{\omega}) & =-\frac{1}{\kappa} h^{-1} d_{h} F \\
\int_{0}^{2 \pi} d x\left(h^{-1} Y^{F}(h)\right)^{0} & =\frac{2 \pi}{\kappa} d_{\omega} F .
\end{aligned}
$$


Given $d_{h} F$ and $d_{\omega} F$, we will next find $b^{-1} Y^{F}(b)$.

On account of (2.8), (2.14) is in fact equivalent to

$$
\left(b^{-1} Y^{F}(b)\right)^{\prime}(x)=-\frac{1}{\kappa} e^{-\bar{\omega} x}\left(h^{-1} d_{h} F\right)(x) e^{\bar{\omega} x},
$$

whose solution is given by

$$
b^{-1}(x) Y^{F}(b(x))=b^{-1}(0) Y^{F}(b(0))-\frac{1}{\kappa} \int_{0}^{x} d y e^{-\bar{\omega} y}\left(h^{-1} d_{h} F\right)(y) e^{\bar{\omega} y} .
$$

Hence the only nontrivial problem is to determine the initial value

$$
Q_{F}:=b^{-1}(0) Y^{F}(b(0))=h^{-1}(0) Y^{F}(h(0)) .
$$

To this end, note from (2.8) that

$$
Y^{F}(\omega)=e^{\omega} b^{-1}(2 \pi) Y^{F}(b(2 \pi)) e^{-\omega}-b^{-1}(0) Y^{F}(b(0)) .
$$

By using (2.17), the Cartan part of (2.19) requires that

$$
Y^{F}(\omega)=-\frac{1}{\kappa} \int_{0}^{2 \pi} d x\left(h^{-1} d_{h} F\right)^{0}(x),
$$

while the root part of (2.19) gives

$$
e^{-\omega} Q_{F}^{r} e^{\omega}-Q_{F}^{r}=-\frac{1}{\kappa} \int_{0}^{2 \pi} d x e^{-\bar{\omega} x}\left(h^{-1} d_{h} F\right)^{r}(x) e^{\bar{\omega} x}
$$

where $Q_{F}=Q_{F}^{0}+Q_{F}^{r}$ according to (2.2). Then (2.21) completely determines $Q_{F}^{r}$ as

$$
Q_{F}^{r}=\frac{1}{\kappa} \sum_{\alpha \in \Phi} \frac{E_{\alpha}}{1-e^{-\alpha(\omega)}} \int_{0}^{2 \pi} d x e^{-\alpha(\bar{\omega}) x} \operatorname{Tr}\left(\left(h^{-1} d_{h} F\right)(x) E^{\alpha}\right) .
$$

As for the remaining unknown, $Q_{F}^{0}$, (2.15) with (2.8) and (2.19) leads to the result:

$$
2 \pi \kappa Q_{F}^{0}=2 \pi d_{\omega} F-\pi \int_{0}^{2 \pi} d x\left(h^{-1} d_{h} F\right)^{0}(x)+\int_{0}^{2 \pi} d x \int_{0}^{x} d y\left(h^{-1} d_{h} F\right)^{0}(y) .
$$

In conclusion, we have found that the hamiltonian vector field $b^{-1} Y^{F}(b)$ is uniquely determined and is explicitly given by (2.17) with $b^{-1}(0) Y^{F}(b(0))=Q_{F}$ in (2.22), (2.23). In the derivation of $Y^{F}$ we have crucially used that $\omega$ is restricted to the domain $\mathcal{A} \subset \mathcal{H}$. At the excluded points of $\mathcal{H}$ some denominators in $(2.22)$ may vanish, whereby $\Omega_{B l o c h}^{G, \kappa}$ becomes singular.

The Poisson bracket of two 'smooth enough' admissible functions $F_{1}$ and $F_{2}$ on $\mathcal{M}_{B l o c h}^{G}$ is determined by the formula

$$
\left\{F_{1}, F_{2}\right\}=Y^{F_{2}}\left(F_{1}\right)=\Omega_{B l o c h}^{G, \kappa}\left(Y^{F_{2}}, Y^{F_{1}}\right) .
$$

We now explain that in a certain sense this Poisson bracket is encoded by the classical exchange algebra (1.3). For this purpose we consider functions of the form

$$
F_{\phi}(h, \omega)=\int_{0}^{2 \pi} d x \operatorname{Tr}\left(\phi(x) b^{\Lambda}(x)\right),
$$


where $b^{\Lambda}(x)$ is taken in a representation $\Lambda$ of $G$ and $\phi(x)$ is a smooth, matrix valued, smearingfunction in that representation. It is easy to check that $F_{\phi}$ is admissible if

$$
\phi^{(k)}(0)=\phi^{(k)}(2 \pi)=0 \quad \forall k=0,1,2 \ldots
$$

and the exterior derivative of $F_{\phi}$ at $(h, \omega)$ is given by

$$
\begin{gathered}
\left(d_{\omega} F_{\phi}\right)(h, \omega)=\frac{1}{2 \pi} \sum_{k} H^{k} \operatorname{Tr}\left(H_{k}^{\Lambda} \int_{0}^{2 \pi} d x\left(x \phi(x) b^{\Lambda}(x)\right)\right) \\
\left(\left(h^{-1} d_{h} F_{\phi}\right)(h, \omega)\right)(x)=\sum_{a} T^{a} \operatorname{Tr}\left(\phi(x) h^{\Lambda}(x) T_{a}^{\Lambda} e^{x \bar{\omega}^{\Lambda}}\right) \quad \text { for } \quad x \in[0,2 \pi] .
\end{gathered}
$$

We here denote by $H_{k}, H^{k}$ and $T_{a}, T^{a}$ dual bases of $\mathcal{H}$ and $\mathcal{G}$, respectively. The last formula extends to a smooth $2 \pi$-periodic function on the real line precisely if (2.26) is satisfied. The hamiltonian vector field $Y^{F_{\phi}}$ is then found to be

$$
\left(b^{-1} Y^{F_{\phi}}(b)\right)(x)=Q_{F_{\phi}}-\frac{1}{\kappa} \sum_{a} T^{a} \int_{0}^{x} d y \operatorname{Tr}\left(\phi(y) b^{\Lambda}(y) T_{a}^{\Lambda}\right), \quad \text { for } \quad x \in[0,2 \pi],
$$

where $Q_{F_{\phi}}$ is determined as described above. By combining the preceding formulae, one can verify that

$$
\left\{F_{\chi}, F_{\phi}\right\}=\Omega_{B l o c h}^{G, \kappa}\left(Y^{F_{\phi}}, Y^{F_{\chi}}\right)=\int_{0}^{2 \pi} \int_{0}^{2 \pi} d x d y \operatorname{Tr}_{12}\left(\chi(x) \otimes \phi(y)\left\{b^{\Lambda}(x) \stackrel{\otimes}{,} b^{\Lambda}(y)\right\}\right)
$$

holds for any $\phi, \chi$ subject to (2.26) provided that one has

$$
\left\{b^{\Lambda}(x) \stackrel{\otimes}{,} b^{\Lambda}(y)\right\}=\frac{1}{\kappa}\left(b^{\Lambda}(x) \otimes b^{\Lambda}(y)\right)\left(\mathcal{R}(\omega)+\frac{1}{2} \hat{I} \operatorname{sign}(y-x)\right)^{\Lambda}, \quad 0<x, y<2 \pi
$$

with $\mathcal{R}(\omega)$ given by (1.4). Since the representation $\Lambda$ is arbitrary, this can be symbolically written in the form (1.3). It is clear that the local formula (1.3) completely encodes the Poisson brackets since $Y^{F_{\phi}}$ can be recovered if the right hand side of $(2.30)$ is given.

Since the current $J=\kappa b^{\prime} b^{-1}$ and the monodromy parameter $\omega$ are functions of $b$, their Poisson brackets can be derived from the exchange algebra (1.3). We can also determine the hamiltonian vector fields of the functions $\omega_{k}:=\operatorname{Tr}\left(\omega H_{k}\right)$ and $\mathcal{F}_{\mu}:=\int_{0}^{2 \pi} d x \operatorname{Tr}(\mu(x) J(x))$, where $\mu$ is a $2 \pi$-periodic, smooth, $\mathcal{G}$-valued test function, directly from (2.14), (2.15) as

$$
Y^{\omega_{k}}(b(x))=\frac{1}{\kappa} b(x) H_{k}, \quad Y^{\mathcal{F}_{\mu}}(b(x))=\mu(x) b(x) .
$$

This implies that $J$ generates the KM Poisson brackets (1.6), and the current algebra is centralized by the functions of $\omega$.

It is worth remarking that the Jacobi identity of the Poisson bracket (1.3) is equivalent to the following equation for the exchange r-matrix:

$$
\left[\mathcal{R}_{12}(\omega), \mathcal{R}_{23}(\omega)\right]+\sum_{k} H_{1}^{k} \frac{\partial}{\partial \omega^{k}} \mathcal{R}_{23}(\omega)+\text { cycl. perm. }=-\frac{1}{4} f_{a b}{ }^{c} T^{a} \otimes T^{b} \otimes T_{c}
$$

\footnotetext{
${ }^{1}$ We use the notation $\operatorname{Tr}=c_{\Lambda} \operatorname{tr}_{\Lambda}$, where $\operatorname{tr}_{\Lambda}$ is the trace over the representation $\Lambda$ and $c_{\Lambda}$ is a normalization factor that makes $c_{\Lambda} \operatorname{tr}\left(\mathrm{A}^{\Lambda} \mathrm{B}^{\Lambda}\right)$ independent of $\Lambda$ for $A, B \in \mathcal{G}$.
} 
where $\left[T_{a}, T_{b}\right]=f_{a b}{ }^{c} T_{c}$ and $\operatorname{Tr}\left(T_{a} T^{b}\right)=\delta_{a}^{b}$. This is a dynamical generalization of the modified classical Yang-Baxter equation, and it has been verified in [1] that $\mathcal{R}(\omega)$ in (1.4) satisfies it. This equation appears in other contexts as well [27, 28, 29] and was recently studied e.g. in [30, 31, 32.

The classical exchange algebra (1.3) is also valid for a compact simple Lie group, $K$, obtained as a real form of a complex simple Lie group, $G$ (like $K=S U(n) \subset S L(n, \mathbf{C})=G$ ). To see this, let $\mathcal{K} \subset \mathcal{G}$ be a compact real form of a complex simple Lie algebra $\mathcal{G}$. One can realize $\mathcal{K}$ as

$$
\mathcal{K}=\operatorname{span}_{\mathbf{R}}\left\{i H_{\alpha_{k}}, F_{\alpha}^{+}, F_{\alpha}^{-} \mid \alpha_{k} \in \Delta, \quad \alpha \in \Phi^{+}\right\}
$$

with

$$
F_{\alpha}^{-}=\frac{|\alpha|}{2}\left(E_{\alpha}-E_{-\alpha}\right), \quad F_{\alpha}^{+}=i \frac{|\alpha|}{2}\left(E_{\alpha}+E_{-\alpha}\right)
$$

where $H_{\alpha_{k}}\left(\alpha_{k} \in \Delta\right), E_{ \pm \alpha}\left(\alpha \in \Phi^{+}\right)$form a Chevalley basis of $\mathcal{G}$ corresponding to the set of simple roots, $\Delta$, and positive roots, $\Phi^{+}$(see e.g. [33]). If $\mathcal{A}$ is now a regular domain in the Cartan subalgebra of $\mathcal{K}$, we have

$$
\mathcal{A} \ni \omega=i \tau=i \sum_{k} \tau^{k} H_{\alpha_{k}} \quad \text { with } \quad \tau^{k} \in \mathbf{R} .
$$

Using also that $\cot (y)=i \operatorname{coth}(i y), \mathcal{R}(\omega)$ in (1.4) can be rewritten as

$$
\mathcal{R}(i \tau)=\sum_{\alpha \in \Phi^{+}} \frac{|\alpha|^{2}}{4} \operatorname{coth}\left(i \frac{1}{2} \alpha(\tau)\right) E_{\alpha} \wedge E_{-\alpha}=\frac{1}{2} \sum_{\alpha \in \Phi^{+}} \cot \left(\frac{1}{2} \alpha(\tau)\right) F_{\alpha}^{+} \wedge F_{\alpha}^{-} .
$$

This means that if $\omega=i \tau$, then the r-matrix on the right hand side of (1.3) lies in $\mathcal{K} \otimes \mathcal{K}$. Therefore (1.3) with (1.4) can be consistently applied to $K$-valued Bloch waves. This can be shown to be the correct result in the compact case by deriving the exchange algebra similarly

as above starting with $\Omega_{B l o c h}^{K, \kappa}$. However, the free field realizations discussed subsequently are valid only in the case of a complex Lie algebra and its normal real form.

\section{Generalized Wakimoto realizations}

At the classical level the generalized Wakimoto realizations of the current algebra are given by certain Poisson maps

$$
W:\left(\widehat{\mathcal{G}}_{0}\right)_{\kappa}^{*} \times T^{*} \widetilde{G}_{+} \longrightarrow(\widehat{\mathcal{G}})_{\kappa}^{*} .
$$

Here $(\widehat{\mathcal{G}})_{\kappa}^{*}$ denotes the space of $\mathcal{G}$-valued currents,

$$
(\widehat{\mathcal{G}})_{\kappa}^{*}=\left\{J \in C^{\infty}(\mathbf{R}, \mathcal{G}) \mid J(x+2 \pi)=J(x)\right\},
$$

equipped with the Poisson bracket in (1.6). The other notations are explained below. By elaborating the idea outlined in [26], we will then prove that $W$ can be lifted to a Poisson (in fact, symplectic) map

$$
\widehat{W}: \mathcal{M}_{\text {Bloch }}^{G_{0}} \times T^{*} \widetilde{G}_{+} \longrightarrow \mathcal{M}_{\text {Bloch }}^{G},
$$

which gives a realization of $G$-valued Bloch waves in terms of $G_{0}$-valued Bloch waves and free fields. 
Let $\mathcal{G}=\sum_{n \in \mathbf{Z}} \mathcal{G}_{n}$ be an integral gradation of $\mathcal{G}$. Define $\mathcal{G}_{+}:=\oplus_{n>0} \mathcal{G}_{n}, \mathcal{G}_{-}:=\oplus_{n<0} \mathcal{G}_{n}$. Denote by $G_{0, \pm} \subset G$ the connected subgroups corresponding to $\mathcal{G}_{0, \pm}$. One can associate a map $W$ (3.1) with the parabolic subalgebra $\left(\mathcal{G}_{+}+\mathcal{G}_{0}\right) \subset \mathcal{G}$ as follows. One of the constituents is the space

$$
\left(\widehat{\mathcal{G}}_{0}\right)_{\kappa}^{*}=\left\{i_{0} \in C^{\infty}\left(\mathbf{R}, \mathcal{G}_{0}\right) \mid i_{0}(x+2 \pi)=i_{0}(x)\right\}
$$

with the Poisson bracket

$$
\left\{\operatorname{Tr}\left(t_{k} i_{0}\right)(x), \operatorname{Tr}\left(t_{l} i_{0}\right)(y)\right\}=\operatorname{Tr}\left(\left[t_{k}, t_{l}\right] i_{0}\right)(x) \delta(x-y)+\kappa \operatorname{Tr}\left(t_{k} t_{l}\right) \delta^{\prime}(x-y),
$$

where $t_{k}$ denotes a basis of $\mathcal{G}_{0}$ and $\operatorname{Tr}$ is the restriction of the scalar product of $\mathcal{G}$ to $\mathcal{G}_{0}$. To describe the other factor in (3.1), consider the manifolds $\widetilde{G}_{+}$and $\widetilde{\mathcal{G}}_{-}$whose elements are smooth, $2 \pi$-periodic functions on $\mathbf{R}$ with values in $G_{+}$and $\mathcal{G}_{-}$, respectively. By means of left translations, identify the cotangent bundle of $\widetilde{G}_{+}$as

$$
T^{*} \widetilde{G}_{+}=\widetilde{G}_{+} \times \widetilde{\mathcal{G}}_{-}=\left\{\left(\eta_{+}, i_{-}\right) \mid \eta_{+} \in \widetilde{G}_{+}, \quad i_{-} \in \widetilde{\mathcal{G}}_{-}\right\} .
$$

The canonical symplectic form on $T^{*} \widetilde{G}_{+}$is given by

$$
\Omega_{T^{*} \widetilde{G}_{+}}=-d \int_{0}^{2 \pi} d x \operatorname{Tr}\left(i_{-} \eta_{+}^{-1} d \eta_{+}\right)
$$

and the corresponding Poisson brackets are encoded by

$$
\begin{aligned}
& \left\{\operatorname{Tr}\left(V^{\alpha} i_{-}\right)(x), \operatorname{Tr}\left(V^{\beta} i_{-}\right)(y)\right\}=\operatorname{Tr}\left(\left[V^{\alpha}, V^{\beta}\right] i_{-}\right)(x) \delta(x-y), \\
& \left\{\operatorname{Tr}\left(V^{\alpha} i_{-}\right)(x), \eta_{+}(y)\right\}=\eta_{+}(x) V^{\alpha} \delta(x-y), \quad\left\{\eta_{+}(x) \stackrel{\otimes}{,} \eta_{+}(y)\right\}=0,
\end{aligned}
$$

where $V^{\alpha}$ is a basis of $\mathcal{G}_{+}$. The map $W$ is defined by the formula:

$$
W:\left(\widehat{\mathcal{G}}_{0}\right)_{\kappa}^{*} \times T^{*} \widetilde{G}_{+} \ni\left(i_{0}, \eta_{+}, i_{-}\right) \mapsto J=\eta_{+}\left(i_{0}-i_{-}\right) \eta_{+}^{-1}+\kappa \eta_{+}^{\prime} \eta_{+}^{-1} \in(\widehat{\mathcal{G}})_{\kappa}^{*} .
$$

One can verify 25] that this is a Poisson map, i.e., the Poisson bracket of $J$ in (1.6) follows from the Poisson brackets of the constituents $\left(i_{0}, \eta_{+}, i_{-}\right)$.

Our purpose now is to complete the construction of the following commutative diagram:

$$
\begin{aligned}
& \mathcal{M}_{\text {Bloch }}^{G_{0}} \times T^{*} \widetilde{G}_{+} \stackrel{\widehat{W}}{\Longrightarrow} \mathcal{M}_{\text {Bloch }}^{G} \\
& \mathcal{D}_{0} \times \text { id } \downarrow \quad \downarrow \mathcal{D} \\
& \left(\widehat{\mathcal{G}_{0}}\right)_{\kappa}^{*} \times T^{*} \widetilde{G}_{+} \stackrel{W}{\longrightarrow}(\widehat{\mathcal{G}})_{\kappa}^{*}
\end{aligned}
$$

The map $\mathcal{D}$ operates according to $\mathcal{D}: b \longmapsto J=\kappa b^{\prime} b^{-1} \cdot \mathcal{M}_{\text {Bloch }}^{G_{0}}$ is the space of $G_{0}$-valued Bloch waves with regular, diagonal monodromy,

$$
\mathcal{M}_{\text {Bloch }}^{G_{0}}=\left\{\eta_{0} \in C^{\infty}\left(\mathbf{R}, G_{0}\right) \mid \eta_{0}(x+2 \pi)=\eta_{0}(x) e^{\omega}, \quad \omega \in \mathcal{A} \subset \mathcal{H}\right\}
$$

with the symplectic form

$$
\Omega_{\text {Bloch }}^{G_{0}, \kappa}\left(\eta_{0}\right)=-\frac{\kappa}{2} \int_{0}^{2 \pi} d x \operatorname{Tr}\left(\eta_{0}^{-1} d \eta_{0}\right) \wedge\left(\eta_{0}^{-1} d \eta_{0}\right)^{\prime}-\frac{\kappa}{2} \operatorname{Tr}\left(\left(\eta_{0}^{-1} d \eta_{0}\right)(0) \wedge d \omega\right) .
$$


Since the same domain $\mathcal{A}$ is used in (2.3) and (3.11), $\mathcal{M}_{B l o c h}^{G_{0}}$ is a symplectic submanifold of $\mathcal{M}_{\text {Bloch }}^{G}$. The map $\mathcal{D}_{0}$ sends $\eta_{0}$ to $i_{0}=\kappa \eta_{0}^{\prime} \eta_{0}^{-1} \in\left(\widehat{\mathcal{G}}_{0}\right)_{\kappa}^{*}$. We have seen that the simple arrows in (3.10) are Poisson maps. The formula of the missing map

$$
\widehat{W}: \mathcal{M}_{\text {Bloch }}^{G_{0}} \times T^{*} \widetilde{G}_{+} \ni\left(\eta_{0}, \eta_{+}, i_{-}\right) \longmapsto b \in \mathcal{M}_{\text {Bloch }}^{G}
$$

can be found from the equation $\mathcal{D} \circ \widehat{W}=W \circ\left(\mathcal{D}_{0} \times\right.$ id $)$, which requires that

$$
\kappa b^{\prime} b^{-1}=\eta_{+}\left(\kappa \eta_{0}^{\prime} \eta_{0}^{-1}-i_{-}\right) \eta_{+}^{-1}+\kappa \eta_{+}^{\prime} \eta_{+}^{-1} .
$$

A solution for $b$ exists that admits a generalized Gauss decomposition. In fact,

$$
b(x)=b_{+}(x) b_{0}(x) b_{-}(x) \quad \text { with } \quad b_{ \pm, 0}(x) \in G_{ \pm, 0}
$$

is a solution of (3.14) if

$$
b_{+}=\eta_{+}, \quad b_{0}=\eta_{0} \quad \text { and } \quad \kappa b_{-}^{\prime} b_{-}^{-1}=-\eta_{0}^{-1} i_{-} \eta_{0} .
$$

The general solution of the differential equation for $b_{-}$can be written in terms of the particular solution $b_{-}^{P}$, defined by $b_{-}^{P}(0)=\mathbf{1}$ as $b_{-}(x)=b_{-}^{P}(x) S$ with an arbitrary $S \in G_{-}$. (Note that $b_{-}^{P}(x)$ is nothing but the path ordered exponential integral of $-\eta_{0}^{-1} i_{-} \eta_{0} / \kappa$ over $[0, x]$.) The constant $S=b_{-}(0)$ has to be determined from the condition that $b$ should have diagonal monodromy. One finds that $b$ has diagonal monodromy, indeed it satisfies $b(x+2 \pi)=b(x) e^{\omega}$, if and only if

$$
e^{-\omega} S e^{\omega}=b_{-}^{P}(2 \pi) S .
$$

Inspecting this equation grade by grade using a parametrization $S=e^{s}, s \in \mathcal{G}_{-}$and the fact that $\omega \in \mathcal{A}$, it is not difficult to see that it has a unique solution for $S$ as a function of $\omega$ and $b_{-}^{P}(2 \pi)$. Determining $S$ in the above manner, we now define the map

$$
\widehat{W}: \mathcal{M}_{\text {Bloch }}^{G_{0}} \times T^{*} \widetilde{G}_{+} \ni\left(\eta_{0}, \eta_{+}, i_{-}\right) \longmapsto b=\eta_{+} \eta_{0} b_{-}^{P} S \in \mathcal{M}_{\text {Bloch }}^{G}
$$

that makes the diagram in (3.10) commutative.

The main result of this paper is the following statement: The map $\widehat{W}$ defined in (3.18) is symplectic, that is,

$$
\left(\widehat{W}^{*} \Omega_{\text {Bloch }}^{G, \kappa}\right)\left(\eta_{0}, \eta_{+}, i_{-}\right)=\Omega_{\text {Bloch }}^{G, \kappa}\left(b=\eta_{+} \eta_{0} b_{-}^{P} S\right)=\Omega_{\text {Bloch }}^{G_{0}, \kappa}\left(\eta_{0}\right)+\Omega_{T^{*} \widetilde{G}_{+}}\left(\eta_{+}, i_{-}\right) .
$$

To prove this, let us restrict the symplectic form $\Omega_{\text {Bloch }}^{G, \kappa}$ in 2.4 to the domain of $\mathcal{M}_{\text {Bloch }}^{G}$ whose elements are decomposable in the form

$$
\begin{gathered}
b=b_{+} b_{0} b_{-} \quad \text { with } \quad b_{0, \pm} \in C^{\infty}\left(\mathbf{R}, G_{0, \pm}\right), \\
b_{+}(x+2 \pi)=b_{+}(x), \quad b_{0}(x+2 \pi)=b_{0}(x) e^{\omega}, \quad b_{-}(x+2 \pi)=e^{-\omega} b_{-}(x) e^{\omega} .
\end{gathered}
$$

A straightforward calculation that uses partial integration and standard properties of the trace yields that in this domain

$$
\begin{aligned}
& \Omega_{\text {Bloch }}^{G, \kappa}(b)=-\frac{\kappa}{2} \int_{0}^{2 \pi} d x \operatorname{Tr}\left(b_{0}^{-1} d b_{0} \wedge\left(b_{0}^{-1} d b_{0}\right)^{\prime}+2\left(b_{0} b_{-}^{\prime} b_{-}^{-1} b_{0}^{-1}\right)\left(b_{+}^{-1} d b_{+} \wedge b_{+}^{-1} d b_{+}\right)\right) \\
& +\kappa \int_{0}^{2 \pi} d x \operatorname{Tr}\left(b_{0} b_{-}\left(b_{-}^{-1} d b_{-}\right)^{\prime} b_{-}^{-1} b_{0}^{-1} \wedge b_{+}^{-1} d b_{+}+\left[d b_{0} b_{0}^{-1}, b_{0} b_{-}^{\prime} b_{-}^{-1} b_{0}^{-1}\right] \wedge b_{+}^{-1} d b_{+}\right) \\
& -\frac{\kappa}{2} \operatorname{Tr}\left(\left(b_{0}^{-1} d b_{0}\right)(0) \wedge d \omega\right)-\frac{\kappa}{2} \operatorname{Tr}\left(\left(b_{-}^{-1} b_{0}^{-1}\left(b_{+}^{-1} d b_{+}\right) b_{0} b_{-}\right)(0) \wedge d \omega\right) \\
& -\frac{\kappa}{2}\left[\operatorname{Tr}\left(b_{-}^{-1} d b_{-} \wedge b_{-}^{-1} b_{0}^{-1}\left(b_{+}^{-1} d b_{+}\right) b_{0} b_{-}\right)\right]_{0}^{2 \pi} .
\end{aligned}
$$


The last two terms cancel, and combining also the other terms we get

$$
\begin{aligned}
\Omega_{\text {Bloch }}^{G, \kappa}(b) & =-\frac{\kappa}{2} \int_{0}^{2 \pi} d x \operatorname{Tr}\left(b_{0}^{-1} d b_{0}\right) \wedge\left(b_{0}^{-1} d b_{0}\right)^{\prime}-\frac{\kappa}{2} \operatorname{Tr}\left(\left(b_{0}^{-1} d b_{0}\right)(0) \wedge d \omega\right) \\
& -d \int_{0}^{2 \pi} d x \operatorname{Tr}\left(\left(-\kappa b_{0} b_{-}^{\prime} b_{-}^{-1} b_{0}^{-1}\right) b_{+}^{-1} d b_{+}\right) .
\end{aligned}
$$

The definition of $\widehat{W}$ says that the image of $\left(\eta_{0}, \eta_{+}, i_{-}\right) \in \mathcal{M}_{\text {Bloch }}^{G_{0}} \times T^{*} \widetilde{G}_{+}$is $b \in \mathcal{M}_{\text {Bloch }}^{G}$ that has the form (3.20) with $b_{0}=\eta_{0}, b_{+}=\eta_{+}$and $b_{-}$specified by the monodromy condition in (3.21) together with $-\kappa \eta_{0} b_{-}^{\prime} b_{-}^{-1} \eta_{0}^{-1}=i_{-}$. Taking this into account, the last equation immediately implies that

$$
\left(\widehat{W}^{*} \Omega_{B l o c h}^{G, \kappa}\right)\left(\eta_{0}, \eta_{+}, i_{-}\right)=\Omega_{B l o c h}^{G_{0}, \kappa}\left(\eta_{0}\right)-d \int_{0}^{2 \pi} d x \operatorname{Tr}\left(i_{-} \eta_{+}^{-1} d \eta_{+}\right),
$$

as required by (3.19).

Since a symplectic map is always a Poisson map as well, $\widehat{W}$ provides us with a realization of the monodromy dependent exchange algebra (1.3) of the $G$-valued Bloch waves in terms of the analogous exchange algebra of the $G_{0}$-valued Bloch waves and the Poisson algebra of $T^{*} \widetilde{G}_{+}$ given by (3.8). Of course, $T^{*} \widetilde{G}_{+}$can also be parametrized by canonical free fields. For this, consider some global coordinates $q^{\alpha}$ on $G_{+}$and define the matrix $\mathcal{N}_{\alpha \beta}(q)=\operatorname{Tr}\left(V_{\beta} \eta_{+}^{-1} \frac{\partial \eta_{+}}{\partial q^{\alpha}}\right)$, where $V_{\beta}$ is a basis of $\mathcal{G}_{-}$dual to the basis $V^{\alpha}$ of $\mathcal{G}_{+}$. By introducing $2 \pi$-periodic canonical free fields $q^{\alpha}(x), p_{\beta}(y)$

$$
\left\{q^{\alpha}(x), p_{\beta}(y)\right\}=\delta_{\beta}^{\alpha}, \quad\left\{q^{\alpha}(x), q^{\beta}(y)\right\}=\left\{p_{\alpha}(x), p_{\beta}(y)\right\}=0,
$$

the Poisson brackets in (3.8) are realized by $\eta_{+}(x)=\eta_{+}(q(x))$ and

$$
i_{-}(x)=-\sum_{\alpha \beta}\left(\mathcal{N}^{-1}\right)^{\alpha \beta}(q) p_{\beta} V_{\alpha},
$$

whereby $\Omega_{T^{*} \widetilde{G}_{+}}=\int_{0}^{2 \pi} d x\left(d p_{\alpha} \wedge d q^{\alpha}\right)$. The map $\widehat{W}$ gives a true free field realization in the principal case, for which $\mathcal{G}_{0}=\mathcal{H}$ is Abelian. In this case $\eta_{0}$ is the exponential of a $\mathcal{H}$-valued free scalar field, $\Psi(x)$, i.e., $\eta_{0}(x)=e^{\Psi(x)}$ with $\Psi(x+2 \pi)=\Psi(x)+\omega$ and

$$
\left\{\operatorname{Tr}\left(H_{k} \Psi\right)(x), \operatorname{Tr}\left(H_{l} \Psi\right)(y)\right\}=\frac{1}{2 \kappa} \operatorname{Tr}\left(H_{k} H_{l}\right) \operatorname{sign}(y-x) \quad \text { for } \quad 0<x, y<2 \pi .
$$

For $G=S L(2)$ the Wakimoto realization of Bloch waves was already described in [7, 15] and some other special cases can be found in [24]. These results are in agreement with our general construction of the map $\widehat{W}$ in (3.18).

Finally, we illustrate the formula in (3.18) by a series of simple examples for the group $G=S L(n)$ (either real or complex). The parabolic subalgebras of $\operatorname{sl}(n)$ are associated with the partitions of $n$, and we consider the 2-block cases (the map $W$ in (3.1) is described in these cases in 25]). That is to say, we let the underlying integral gradation of $\mathcal{G}=\operatorname{sl}(n)$ be defined by the eigenvalues of $\operatorname{ad} Q$ with a diagonal matrix

$$
Q=\frac{1}{n} \operatorname{diag}\left(n_{2} \mathbf{1}_{n_{1}},-n_{1} \mathbf{1}_{n_{2}}\right), \quad n=n_{1}+n_{2} .
$$


In this case $\mathcal{G}_{ \pm}$are Abelian subalgebras, which leads to a simplification of the formulae. In particular, we can introduce the convenient parametrizations

$$
\eta_{+}=\left[\begin{array}{cc}
\mathbf{1}_{n_{1}} & q \\
0 & \mathbf{1}_{n_{2}}
\end{array}\right], \quad i_{-}=-\left[\begin{array}{cc}
\mathbf{0}_{n_{1}} & 0 \\
p & \mathbf{0}_{n_{2}}
\end{array}\right]
$$

where $q(x)$ and $p(x)$ are $n_{1} \times n_{2}$ and $n_{2} \times n_{1}$ matrices, whose entries satisfy

$$
\left\{q_{a b}(x), p_{c d}(y)\right\}=\delta_{a d} \delta_{b c} \delta(x-y) .
$$

In terms of the parametrizations

$$
\eta_{0}=\left[\begin{array}{cc}
\eta_{u} & 0 \\
0 & \eta_{d}
\end{array}\right], \quad b_{-}^{P}=\left[\begin{array}{cc}
\mathbf{1}_{n_{1}} & 0 \\
B & \mathbf{1}_{n_{2}}
\end{array}\right]
$$

the solution of the differential equation in (3.16) is then found to be

$$
B(x)=\frac{1}{\kappa} \int_{0}^{x} d y\left(\eta_{d}^{-1} p \eta_{u}\right)(y) .
$$

Furthermore, if we now denote

$$
S=\left[\begin{array}{cc}
\mathbf{1}_{n_{1}} & 0 \\
\sigma & \mathbf{1}_{n_{2}}
\end{array}\right], \quad \omega=\operatorname{diag}\left(\omega_{1}, \omega_{2}, \ldots, \omega_{n}\right),
$$

then eq. (3.17) is explicitly solved as

$$
\sigma_{a b}=\frac{B_{a b}(2 \pi)}{\exp \left(\omega_{b}-\omega_{a+n_{1}}\right)-1}, \quad 1 \leq a \leq n_{2}, \quad 1 \leq b \leq n_{1} .
$$

Combining these equations, (3.18) yields a realization of the $S L(n)$-valued Bloch-wave $b$ in terms of the fields $q, p$ and $\eta_{0}$. By subsequently using a similar Wakimoto realization for $\eta_{0}$, and so on, one can iteratively build up a complete free field realization of $b$. Incidentally, $\eta_{0}$ can be written in the alternative form

$$
\eta_{0}=e^{Q \varphi}\left[\begin{array}{cc}
\tilde{\eta}_{u} & 0 \\
0 & \tilde{\eta}_{d}
\end{array}\right]
$$

where $e^{Q \varphi(x)}, \tilde{\eta}_{u}(x)$ and $\tilde{\eta}_{d}(x)$ are $U(1), S L\left(n_{1}\right)$ and $S L\left(n_{2}\right)$-valued independent Bloch-waves.

The Wakimoto realizations of the affine KM algebras in generalized Fock spaces, at the level of vertex algebras as opposed to the above Poisson algebras, have many applications in conformal field theory [2, 22, 23]. An explicit formula for such realizations of the current $J$ was derived in [25] by quantizing the expression (3.9). It would be very interesting to also quantize (3.18). The exchange algebra of the resulting vertex operators should contain the quantized version of the r-matrix $\mathcal{R}(\omega)$ which has been constructed recently for all Lie algebras in a universal manner [34. Another open problem is to find an analogue of the rather simple construction of the Wakimoto realizations presented here and in [25] for the case of $q$-deformed affine KM algebras. We hope to return to these questions in the future.

Acknowledgements. This investigation was supported in part by the Hungarian National Science Fund (OTKA) under T019917, T030099, T025120 and by the Ministry of Education under FKFP 0178/1999 and FKFP 0596/1999. 


\section{References}

[1] E. Witten, Commun. Math. Phys. 92 (1984) 455.

[2] P. Di Francesco, P. Mathieu and D. Sénéchal, Conformal Field Theory (Springer, 1996).

[3] P. Etingof, I. Frenkel and A. Kirillov Jr., Lectures on Representation Theory and KnizhnikZamolodchikov Equations (AMS, 1998).

[4] O. Babelon, Phys. Lett. B 215 (1988) 523.

[5] B. Blok, Phys. Lett. B 233 (1989) 359.

[6] L. Faddeev, Commun. Math. Phys. 132 (1990) 131.

[7] A. Alekseev and S. Shatashvili, Commun. Math. Phys. 133 (1990) 353.

[8] T. Itoh and Y. Yamada, Prog. Theor. Phys. 85 (1991) 751.

[9] V.A. Fateev and S.L. Lukyanov, Int. J. Mod. Phys. A 7 (1992) 853.

[10] A. Alekseev and I.T. Todorov, Nucl. Phys. B 421 (1994) 413.

[11] J. Balog, L. Dąbrowski and L. Fehér, Phys. Lett. B 244 (1990) 227.

[12] M. Chu, P. Goddard, I. Halliday, D. Olive and A. Schwimmer, Phys. Lett. B 266 (1991) 71.

[13] O. Babelon, F. Toppan and L. Bonora, Commun. Math. Phys. 140 (1991) 93.

[14] K. Gawędzki, Commun. Math. Phys. 139 (1991) 201.

[15] F. Falceto and K. Gawędzki, J. Geom. Phys. 11 (1993) 251.

[16] J. Balog, L. Fehér and L. Palla, Phys. Lett. B 463 (1999) 83 (to appear), hep-th/9907050; Chiral extensions of the WZNW phase space, Poisson-Lie symmetries and groupoids, hep-th/9910046.

[17] C. Gómez, M. Ruiz-Altaba and G. Sierra, Quantum Groups in Two-Dimensional Physics (Cambridge University Press, 1996), and references therein.

[18] M. Chu and P. Goddard, Phys. Lett. B 337 (1994) 285; Nucl. Phys. B 445 (1995) 145.

[19] P. Furlan, L.K. Hadjiivanov and I. T. Todorov, Nucl.Phys. B 474 (1996) 497; Int. J. Mod. Phys. A 12 (1997) 23.

[20] L. Caneschi and M. Lysiansky, Nucl. Phys. B 505 (1997) 701.

[21] M. Wakimoto, Commun. Math. Phys. 104 (1986) 605.

[22] B.L. Feigin and E.V. Frenkel, Russ. Math. Surv. 43 (1989) 221; Commun. Math. Phys. 128 (1990) 161;

E. Frenkel, Free field realizations in representation theory and conformal field theory, hep-th/9408109.

[23] P. Bouwknegt, J. McCarthy and K. Pilch, Commun. Math. Phys. 131 (1990) 125; Prog. Theor. Phys. Suppl. 102 (1990) 67. 
[24] A. Gerasimov, A. Morozov, M. Olshanetsky, A. Marshakov and S. Shatashvili, Int. J. Mod. Phys. A5 (1990) 2495.

[25] J. de Boer and L. Fehér, Mod. Phys. Lett. A 11 (1996) 1999; Commun. Math. Phys. 189 (1997) 759 .

[26] L. Fehér, in: Proc. of the 7th Colloquium on Quantum Groups and Integrable Systems, Czech. J. Phys. 48 (1998) 1325.

[27] J.-L. Gervais and A. Neveu, Nucl. Phys. B 238 (1984) 125;

E. Cremmer and J.-L. Gervais, Commun. Math. Phys. 134 (1990) 619.

[28] G. Felder and C. Wieczerkowski, Commun. Math. Phys. 176 (1996) 133;

G. Felder, pp. 1247-1255 in: Proc. Int. Congr. Math. Zürich, 1994 (hep-th/9407154).

[29] J. Avan, O. Babelon and E. Billey, Commun. Math. Phys. 178 (1996) 281.

[30] P. Etingof and A. Varchenko, Commun. Math. Phys. 192 (1998) 77.

[31] Z. J. Liu and P. Xu, Dirac structures and dynamical r-matrices, math.DG/9903119.

[32] J.H. Lu, Classical dynamical r-matrices and homogeneous Poisson structures on $G / H$ and $K / T$, math/9909004.

[33] V.V. Gorbatsevich, A.L. Onishchik and E.B. Vinberg, Structure of Lie Groups and Lie Algebras, Encyclopaedia of Mathematical Sciences, Vol. 41 (Springer, 1994).

[34] D. Arnaudon, E. Buffenoir, E. Ragoucy and Ph. Roche, Lett. Math. Phys. 44 (1998) 201. 